\title{
Possible causes of variation in acrylamide concentration in French fries prepared in food service establishments: An observational study.
}

\begin{abstract}
Acrylamide is a probable human carcinogen, and its presence in a range of fried and ovencooked foods has raised considerable health concern world-wide. Dietary intake studies observed significant variations in acrylamide concentrations, which complicate risk assessment and the establishment of effective control measures. The objective of this study was to obtain an insight into the actual variation in acrylamide concentrations in French fries prepared under typical conditions in a food service establishment (FSE). Besides acrylamide, frying time, frying temperature, and reducing sugars were measured and the actual practices at receiving, thawing and frying during French fries preparation were observed and recorded. The variation in the actual frying temperature contributed most to the variation in acrylamide concentrations, followed by the variation in actual frying time; no obvious effect of reducing sugars was found. The lack of standardised control of frying temperature and frying time (due to inadequate frying equipment) and the variable practices of food handlers seem to contribute most to the large variation and high acrylamide concentrations in French fries prepared in a restaurant type of FSE as compared to chain fast-food services, and institutional caterers. The obtained insights in this study can be used to develop dedicated control measures in FSE, which may contribute to a sustainable reduction in the acrylamide intake.
\end{abstract}

Keyword: Acrylamide concentration; French fries; Food service establishments. 